\title{
Association between polymorphisms in selected inflammatory response genes and the risk of prostate cancer
}

This article was published in the following Dove Press journal:

OncoTargets and Therapy

II January 2016

Number of times this article has been viewed

\author{
Jun Chen ${ }^{1, *}$ \\ Xue-Ming Ying ${ }^{2, *}$ \\ Xue-Ming Huang ${ }^{3}$ \\ Peng Huang ${ }^{4}$ \\ Shao-Cong Yan'
}

'Department of Oncology, The First Affiliated Hospital of Nanchang University, Nanchang, ${ }^{2}$ Department of Oncology, Jingdezhen City People's Hospital, Jingdezhen, ${ }^{3}$ Department of Urology, Research Institute, The First Affiliated Hospital of Nanchang University, ${ }^{4}$ The Medical School of Nanchang University, School of Public Health, Nanchang, People's Republic of China

*These authors contributed equally to this work
Correspondence: Xue-Ming Ying Department of Oncology, Jingdezhen City People's Hospital, No 317 North Road, Jingdezhen 333000, Jiangxi, People's Republic of China $\mathrm{Tel} / \mathrm{fax}+867988560388$

Email yingxueming63@gmail.com
Abstract: Inflammation represents an important event which facilitates prostate carcinogenesis. Genetic variations in inflammatory response genes could affect the level and function of the protein products, resulting in the differential prostate cancer risk among carriers of different variants. This study attempted to investigate the association of $I L-4 \mathrm{rs} 2243250, I L-6 \mathrm{rs} 10499563$, IL- 8 rs 4073, as well as NFKBIA rs2233406 and rs3138053 polymorphisms with prostate cancer risk in the Chinese population. Genotyping of the polymorphisms was performed by using polymerase chain reaction-restriction fragment length polymorphism technique on 439 prostate cancer patients and 524 controls, and the association of each polymorphic genotype with prostate cancer risk was evaluated by using logistic regression analysis based on allele, heterozygous, and homozygous comparison models, with adjustment to age and smoking status. We showed that the $\mathrm{C}$ allele of $I L-4 \mathrm{rs} 2243250$ polymorphism could increase prostate cancer risk (heterozygous comparison model: odds ratio $[\mathrm{OR}]=1.434,95 \%$ confidence interval $[\mathrm{CI}]=1.092-1.881$, $P=0.009$; homozygous comparison model: $\mathrm{OR}=2.301,95 \% \mathrm{CI}=1.402-3.775, P=0.001$; allele comparison model: $\mathrm{OR}=1.509,95 \% \mathrm{CI}=1.228-1.853, P<0.001)$. On the other hand, the $\mathrm{C}$ allele of rs 10499563 polymorphism could decrease prostate cancer risk (heterozygous comparison model: $\mathrm{OR}=0.694,95 \% \mathrm{CI}=0.525-0.918, P=0.010$; homozygous comparison model: $\mathrm{OR}=0.499,95 \% \mathrm{CI}=0.269-0.926, P=0.028$; allele comparison model: $\mathrm{OR}=0.692$, $95 \% \mathrm{CI}=0.553-0.867, P=0.001)$. No association was observed for the other polymorphisms In conclusion, $I L-4$ rs2243250 and $I L-6$ rs10499563 polymorphisms could serve as potential predictive biomarkers for prostate cancer risk in the Chinese population.

Keywords: biomarkers, inflammation, logistic regression, polymorphisms, prostate neoplasm

\section{Introduction}

Prostate cancer is the second most common form of cancer in the world. ${ }^{1}$ Each year, there is an estimated 914,000 new cases of prostate cancer worldwide. ${ }^{1}$ In the People's Republic of China, although the incidence of prostate cancer is comparatively lower than that in the Western countries, the occurrence of prostate cancer appears to be rapidly increasing in recent years. ${ }^{2}$ The age-standardized incidence rates of prostate cancer in the People's Republic of China increased several folds between 1970s and late 1990 s/early 2000 s, that is, from $5.1 / 100,000$ to $15 / 100,000$ in Hong Kong and from $0.8 / 100,000$ to $6.9 / 100,000$ in Shanghai. ${ }^{3}$ The alarming rate of increase in prostate cancer incidences is a significant public health concern, as prostate cancer accounts for $\sim 10 \%$ of cancer-related mortality in males. ${ }^{4}$

Despite the seriousness of the disease, the etiology of prostate cancer remains poorly understood. It is known, however, that interaction between genetic and environmental 
factors contributes to the disease susceptibility. ${ }^{5}$ In recent years, inflammation has emerged as an important event which facilitates carcinogenesis. ${ }^{6}$ There is increasing epidemiological and biological evidence which suggests the role of chronic inflammation in the prostate, as a result of dysregulation of inflammatory response genes, in prostate

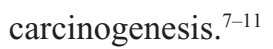

Genetic variations, such as single-nucleotide polymorphisms (SNPs), could influence the transcriptional efficiency of a gene, as well as structure (and hence, function) of the protein product. Therefore, if genetic variations occur in inflammatory response genes, and if inflammation is a risk factor for prostate cancer, those variations which contribute to a high inflammatory level should be able to increase prostate cancer risk and vice versa. Several studies have suggested that although SNPs in low penetrance genes could influence the risk of prostate cancer modestly, they are highly prevalent in the general population compared to mutations in high penetrance genes. ${ }^{12-14}$ Therefore, many researchers have focused on identifying such polymorphic variants in the past decade. ${ }^{12-14}$ The effect of SNPs on cancer risk is known to differ across populations, and report on the association of SNPs in inflammatory response genes with prostate cancer risk in People's Republic of China is currently scarce.

A few SNPs have been commonly implicated with the risk of prostate and/or other cancers. This includes the rs2243250 polymorphism of $I L-4$, rs 10499563 polymorphism of $I L-6$, rs4073 polymorphism of $I L-8$, as well as rs2233406 and rs3138053 polymorphisms of NFKBIA. Notably, these SNPs are all capable of influencing the transcriptional and/or translational activity of their respective genes. Interleukin-4 is a pleiotropic type I cytokine produced by $\mathrm{T}$ helper 2 cells, which plays an anti-inflammatory role and is also a potent inhibitor of angiogenesis. ${ }^{15}$ Interleukin-6 is a pro-inflammatory cytokine which also regulates several carcinogenic events such as apoptosis, angiogenesis, and cell proliferation. Interleukin- 8 is a pro-inflammatory cytokine which has been implicated in a wide variety of carcinogenic processes, such as angiogenesis and invasion or metastasis of cancer. $N F K B I A$ encodes for $\mathrm{I} \kappa \mathrm{B} \alpha$, a protein which serves as an absolute requirement to inactivate the $\mathrm{NF}-\kappa \mathrm{B}$, a class of pleiotropic transcription factors which act as central regulators in many carcinogenic pathways, including inflammation. ${ }^{16}$ Given the important role of these genes in the inflammatory pathway, we hypothesized that polymorphisms in these genes could influence prostate cancer risk. This study attempted to examine the association of rs 2243250 polymorphism of $I L-4$, rs 10499563 polymorphism of $I L-6$, rs4073 polymorphism of $I L-8$, as well as rs2233406 and rs3138053 polymorphisms of NFKBIA with prostate cancer risk in the Chinese population. In addition, since age and tobacco smoking have been established as important risk factors of prostate cancer, ${ }^{17}$ we adjusted our odds ratio (OR) findings according to these parameters.

\section{Materials and methods Patients and controls}

Between July 2010 and July 2013, 439 newly diagnosed prostate cancer patients were recruited from The First Affiliated Hospital of Nanchang University, Jiangxi, People's Republic of China. Diagnosis of prostate cancer was done based on clinical, pathological, and laboratory tests by qualified physicians and laboratory personnel according to the established guideline of prostate cancer diagnosis. All patients had a prostate-specific antigen (PSA) level of $>10 \mathrm{ng} / \mathrm{mL}$ and were without metastasis. In addition, 524 male volunteers who visited the same hospital for general medical checkup (and were proven to be healthy and free from any medical conditions through the medical checkup) were recruited into the present study as controls without cancer. The controls were individuals with a PSA level of $<2 \mathrm{ng} / \mathrm{mL}$. The use of PSA level of $>10 \mathrm{ng} / \mathrm{mL}$ in cases and of $<2 \mathrm{ng} / \mathrm{mL}$ in controls served as a criterion to prevent false positivity and false negativity, respectively. All the subjects were self-described Han Chinese ethnicity and had no prior history of other cancers. Written informed consent was obtained from all participants at recruitment. The study was approved by the Medical Ethics Committee of Nanchang University.

\section{Laboratory analysis}

Peripheral blood was collected from all the participants, and DNA was isolated from the specimens obtained by using Tiangen DNA isolation kit (Tiangen Biotech, Beijing, People's Republic of China). Briefly, $200 \mu \mathrm{L}$ of blood sample was lysed with Proteinase K and Buffer GB, followed by precipitation of DNA using ethanol and further purification through the spin column by using the respective buffers supplied. The DNA was then eluted in Buffer TE. The DNA was used for genotyping of the five SNPs. All the SNPs were genotyped with polymerase chain reaction (PCR)-restriction fragment length polymorphism (RFLP) method, as summarized in Table 1. All temperature controls (in both PCR and RFLP) were performed by using thermocycler. The sequence of the PCR primers and the restriction enzymes used were all obtained from the literature. ${ }^{18-21}$ For each SNP, $\sim 20 \%$ of the 
Table I PCR primer sequence, annealing temperature, and product size, as well as restriction enzyme used and band sizes obtained after digestion, for genotyping of the polymorphisms

\begin{tabular}{|c|c|c|c|c|c|c|}
\hline SNP & Primer sequence & $\begin{array}{l}\text { Annealing } \\
\text { temperature }\left({ }^{\circ} \mathrm{C}\right)\end{array}$ & $\begin{array}{l}\text { PCR product } \\
\text { size (bp) }\end{array}$ & $\begin{array}{l}\text { Restriction } \\
\text { enzyme }\end{array}$ & Genotype (bp) & References \\
\hline rs2243250 & $\begin{array}{l}\text { 5'-TAAACTTGGGAGAACATGGT-3' } \\
\text { 5'-TGGGGAAAGATAGAGTAATA-3' }\end{array}$ & 52 & 195 & Avall & $\begin{array}{l}\text { TT: } 195 \\
\text { CT: } 195+176+19 \\
\text { CC: } 176+19\end{array}$ & 15 \\
\hline rs10499563 & $\begin{array}{l}\text { 5'-AGGGAAAGCAGGTTATCAAA-3' } \\
\text { 5'-AGTGGCTTCAGGGAGACTAA-3' }^{\prime}\end{array}$ & 61 & 424 & Taal & $\begin{array}{l}\text { TT: } 424 \\
\text { TC: } 424+320+104 \\
\text { CC: } 320+104\end{array}$ & 16 \\
\hline rs4073 & $\begin{array}{l}\text { 5'-ATCTTGTTCTAACACCTGCCACTCT-3' } \\
\text { 5'-TAAAATACTGAAGCTCCACAATTTGG-3' }\end{array}$ & 59 & 121 & Munl & $\begin{array}{l}\text { TT: } 121 \\
\text { TA: } 121+82+39 \\
\text { AA: } 82+39\end{array}$ & 17 \\
\hline rs2233406 & $\begin{array}{l}\text { 5'-GGTCCTTAAGGTCCAATCG-3' } \\
\text { 5'-GTTGTGGATACCTTGCACTA-3' }\end{array}$ & 62 & 200 & Bfal & $\begin{array}{l}\text { CC: } 180+20 \\
\text { CT: } 200+180+20 \\
\text { TT: } 200\end{array}$ & 18 \\
\hline rs3138053 & $\begin{array}{l}\text { 5'-GGTCCTTAAGGTCCAATCG-3' } \\
\text { 5'-GTTGTGGATACCTTGCACTA-3' }\end{array}$ & 62 & 200 & TspRI & $\begin{array}{l}\text { AA: } 200 \\
\text { AG: } 200+|29+7| \\
\text { GG: }|29+7|\end{array}$ & 18 \\
\hline
\end{tabular}

Abbreviations: PCR, polymerase chain reaction; SNP, single-nucleotide polymorphism.

samples were chosen randomly and sequenced to reconfirm the genotypes. The concordance rate of the sequencing result and the result of PCR-RFLP genotyping was $99.9 \%$.

\section{Statistical analysis}

SPSS (version 21.0) was used for all statistical analyses. The presence of significant difference between the mean ages of cases and controls was evaluated using Student's $t$-test. Chi-square test was used to determine the difference in the smoking status, as well as the distribution of alleles and genotypes, of the cases and controls. Similarly, chi-square test was used to determine whether the distribution deviates from Hardy-Weinberg equilibrium. Multivariate logistic regression analysis (with adjustment to age and smoking status) was employed to evaluate the association of the genotypes and prostate cancer risk based on heterozygous and homozygous comparison models, with the wild-type genotype serving as the reference. OR of $>1.00$ indicated a positive risk association, and vice versa. $P$-values of $<0.05$ were significant.

\section{Results}

\section{General characteristics}

The general characteristics of the subjects are listed in Table 2 . The cases consisted of individuals aged between 48 and 87 years, and the age range of controls was $42-85$ years. The mean age of cases was $69.85 \pm 8.66$, while the mean age of controls was $69.39 \pm 8.74$. No significant difference was observed between the mean ages of both groups $(P=0.420)$.
For both cases and controls, the frequency of never smokers was slightly higher than ever smokers. In cases, $45.79 \%$ were ever smokers, and $54.21 \%$ were never smokers, while in controls, $44.08 \%$ were ever smokers, and $55.92 \%$ were never smokers. The difference in the distribution of ever and never smokers among cases and controls was not significant $(P=0.597)$.

\section{Distribution of alleles and genotypes}

The distribution of alleles and genotypes of the five polymorphisms in cases and controls is shown in Table 3. For the $I L-4$ rs 2243250 polymorphism, significant difference was observed between cases and controls in the distribution of TT and CC genotypes. TT genotype was overrepresented in controls group ( $P=0.001)$, while CC genotype was overrepresented in the cases group $(P=0.005)$. Significant difference was also seen for the allele distribution, where the $\mathrm{T}$ allele was found at a higher frequency in controls than cases, and vice versa for $\mathrm{C}$ allele $(P<0.001)$. However, no significant difference was observed for the CT genotype $(P=0.056)$.

Table 2 General characteristics of the subjects

\begin{tabular}{llll}
\hline Characteristics & Case $(\mathbf{n = 4 3 9 )}$ & Control $(\mathbf{n = 5 2 4 )}$ & $P$-value \\
\hline Age, years & & & 0.420 \\
$\quad$ Mean \pm SD & $69.85 \pm 8.66$ & $69.39 \pm 8.74$ & \\
$\quad$ Range & $48-87$ & $42-85$ & \\
Smoking status & & & 0.597 \\
$\quad$ Ever smokers & $201(45.79 \%)$ & $231(44.08 \%)$ & \\
$\quad$ Never smokers & $238(54.21 \%)$ & $293(55.92 \%)$ & \\
\hline
\end{tabular}

Abbreviation: SD, standard deviation. 
Table 3 Genotype and allele distribution of the five polymorphisms, as well as Hardy-Weinberg equilibrium of the controls

\begin{tabular}{|c|c|c|c|c|}
\hline & Case $(n=439), n(\%)$ & Control $(n=524), n(\%)$ & $P$-value ${ }^{a}$ & HWE $_{\text {control }}$ \\
\hline \multicolumn{5}{|l|}{ rs2243250 } \\
\hline TT & $222(50.6)$ & $322(61.5)$ & 0.001 & $\chi^{2}=0.810$ \\
\hline TC & $|7|(39.0)$ & $173(33.0)$ & 0.056 & $P=0.360$ \\
\hline CC & $46(10.5)$ & $29(5.5)$ & 0.005 & \\
\hline T allele & $615(70.0)$ & $817(78.0)$ & $<0.001$ & \\
\hline C allele & $263(30.0)$ & $23 I(22.0)$ & & \\
\hline \multicolumn{5}{|l|}{ rsI0499563 } \\
\hline TT & $301(68.6)$ & $310(59.2)$ & 0.003 & $\chi^{2}=0.891$ \\
\hline TC & $122(28.0)$ & 181 (34.5) & 0.025 & $P=0.345$ \\
\hline $\mathrm{CC}$ & $16(3.6)$ & $33(6.3)$ & 0.065 & \\
\hline T allele & $724(82.5)$ & $80 I(76.4)$ & 0.001 & \\
\hline C allele & $154(17.5)$ & $247(23.6)$ & & \\
\hline \multicolumn{5}{|l|}{ rs4073 } \\
\hline TT & $164(37.4)$ & $178(34.0)$ & 0.274 & $\chi^{2}=2.306$ \\
\hline TA & $20 I(45.8)$ & $240(45.8)$ & 0.996 & $P=0.129$ \\
\hline AA & $74(16.9)$ & $106(20.2)$ & 0.182 & \\
\hline $\mathrm{T}$ allele & $529(60.3)$ & $596(56.9)$ & 0.134 & \\
\hline A allele & $349(39.7)$ & $452(43.1)$ & & \\
\hline \multicolumn{5}{|l|}{ rs2233406 } \\
\hline $\mathrm{CC}$ & $319(72.7)$ & $40 \mathrm{I}(76.5)$ & 0.170 & $\chi^{2}=0.379$ \\
\hline CT & $106(24.1)$ & $113(21.6)$ & 0.342 & $P=0.538$ \\
\hline TT & $14(3.2)$ & $10(1.9)$ & 0.209 & \\
\hline$C$ allele & 744 (84.7) & $915(87.3)$ & 0104 & \\
\hline $\mathrm{T}$ allele & $134(15.3)$ & $133(12.7)$ & 0.104 & \\
\hline \multicolumn{5}{|l|}{ rs3138053 } \\
\hline AA & $324(73.8)$ & $398(76.0)$ & 0.443 & $\chi^{2}=1.621$ \\
\hline AG & $109(24.8)$ & $|2|(23.1)$ & 0.529 & $P=0.203$ \\
\hline GG & $6(1.4)$ & $5(1.00)$ & 0.551 & \\
\hline$A$ allele & 757 (86.2) & 917 (87.5) & & \\
\hline G allele & $121(13.8)$ & $|3|(\mid 2.5)$ & 0.406 & \\
\hline
\end{tabular}

Notes: aSignificant at $P<0.05$. Data in bold indicates statistical significance.

Abbreviation: $\mathrm{HWE}_{\text {control' }}$, Hardy-Weinberg equilibrium in controls.

On the other hand, for the $I L-6$ rs 10499563 polymorphism, overrepresentation of the TT genotype was observed in cases $(P=0.003)$, and the TC genotype was overrepresented in controls $(P=0.025)$. Although the prevalence of CC genotype was higher in controls, the difference was not statistically significant $(P=0.065)$. In terms of allele frequency, the distribution of T and $\mathrm{C}$ genotypes was statistically significantly different between cases and controls, with a $P$-value of 0.001 .

For the other three polymorphisms, no statistically significant difference was observed between cases and controls in the allelic and genotypic distributions. For $I L-8$ rs 4073 polymorphism, the TT genotype was slightly overrepresented in cases $(P=0.274)$, and the AA genotype was observed in a slightly higher frequency in controls $(P=0.182)$. The frequency of the TA heterozygous genotypes was almost identical in cases and controls, with a $P$-value of 0.996 . The frequency of $\mathrm{T}$ and $\mathrm{A}$ alleles was not significantly different between cases and controls $(P=0.134)$. For NFKBIA rs2233406 and rs3138053 polymorphisms, the frequency of homozygous wild-type genotype (CC and AA, respectively) was higher in controls, while that of the heterozygous (CT and AG, respectively) and homozygous variant (TT and GG, respectively) was higher in controls. However, the difference did not reach statistical significance. For NFKBIA rs2233406, the $P$-values for CC, CT, and TT genotypes were 0.170, 0.342, and 0.209, respectively, while for NFKBIA rs3138053, the $P$-values for AA, AG, and GG genotypes were $0.443,0.529$, and 0.551 , respectively. The differences in allele distribution were also not statistically significant $(P=0.104$ for NFKBIA rs2233406 and $P=0.406$ for NFKBIA rs3138053).

For all polymorphisms, the distribution of genotypes did not deviate significantly from Hardy-Weinberg equilibrium $(P<0.05)$ (Table 3).

\section{Risk association}

Table 4 shows the association between the five polymorphisms and prostate cancer risk in the population studied. For $I L-4$ rs2243250 polymorphism, a significantly increased 
Table 4 Association of the polymorphisms and prostate cancer risk based on heterozygous, homozygous, and allele comparison models

\begin{tabular}{|c|c|c|c|}
\hline SNP & Comparison model & Odds ratio $(95 \% \mathrm{CI})^{a}$ & $P$-value ${ }^{b}$ \\
\hline \multirow[t]{3}{*}{ rs2243250 } & Heterozygous comparison (TC vs TT) & $\mathrm{I} .430(\mathrm{I} .085, \mathrm{I} .877)$ & 0.010 \\
\hline & Homozygous comparison (CC vs TT) & $2.293(1.397,3.763)$ & 0.001 \\
\hline & Allele comparison (C vs T) & $1.509(1.228,1.853)$ & $<0.001$ \\
\hline \multirow[t]{3}{*}{ rs 10499563} & Heterozygous comparison (TC vs TT) & $0.696(0.526,0.920)$ & 0.011 \\
\hline & Homozygous comparison (CC vs TT) & $0.504(0.272,0.936)$ & 0.030 \\
\hline & Allele comparison (C vs T) & $0.692(0.553,0.867)$ & 0.001 \\
\hline \multirow[t]{3}{*}{ rs4073 } & Heterozygous comparison (TA vs TT) & $0.912(0.687,1.211)$ & 0.523 \\
\hline & Homozygous comparison (AA vs TT) & $0.762(0.529,1.098)$ & 0.145 \\
\hline & Allele comparison (A vs T) & $0.873(0.727,1.047)$ & 0.143 \\
\hline \multirow[t]{3}{*}{ rs2233406 } & Heterozygous comparison (CT vs CC) & I.I88 (0.877, I.6I0) & 0.265 \\
\hline & Homozygous comparison (TT vs CC) & $1.762(0.772,4.02 \mathrm{I})$ & 0.179 \\
\hline & Allele comparison ( $T$ vs $C$ ) & $1.246(0.962,1.614)$ & 0.096 \\
\hline \multirow[t]{3}{*}{ rs3138053 } & Heterozygous comparison (AG vs AA) & $1.121(0.832,1.512)$ & 0.452 \\
\hline & Homozygous comparison (GG vs AA) & $1.501(0.453,4.973)$ & 0.507 \\
\hline & Allele comparison ( $\mathrm{G}$ vs $A$ ) & I.I $32(0.868$, I.476) & 0.361 \\
\hline
\end{tabular}

Notes: aSignificant at $P<0.05$. ${ }^{b}$ Adjusted to age and smoking status.

Abbreviations: SNP, single-nucleotide polymorphism; Cl, confidence interval.

prostate cancer risk was observed in the heterozygous comparison model (TC vs TT) and homozygous comparison model (CC vs TT), after adjustment to age and smoking status. In heterozygous comparison model, the adjusted OR was 1.430 (95\% confidence interval [CI]: 1.085-1.877), with a $P$-value of 0.010 . On the other hand, in homozygous comparison model, the adjusted OR was 2.239 (95\% $\mathrm{CI}=1.397-3.763, P=0.001)$. In allele comparison model, the $\mathrm{C}$ allele was shown to result in an increased risk with an adjusted OR of 1.509 (95\% CI $=1.228-1.853, P<0.001)$.

Similarly, for $I L-6$ rs 10499563 polymorphism, significant risk association was observed in the heterozygous (TC vs TT) and homozygous comparison (CC vs TT) models. However, rather than an increased risk association, $I L-6$ rs10499563 polymorphism showed a reduced prostate cancer risk in the three comparison models. In heterozygous comparison model, the adjusted OR was 0.696 (95\% CI $=0.526-0.920$, $P=0.011$ ), while a greater risk reduction was observed in the homozygous comparison model ( $\mathrm{OR}=0.504,95 \%$ $\mathrm{CI}=0.272-0.936, P=0.030$ ). Besides, in allele comparison model, the $\mathrm{C}$ allele showed an adjusted OR of 0.692 (95\% $\mathrm{CI}=0.553-0.867, P=0.001)$.

Statistically significant prostate cancer risk association was not found for the $I L-8$ rs4073, NFKBIA rs2233406, and NFKBIA rs3138053 polymorphisms in all the three comparison models investigated $(P>0.05)$.

\section{Discussion}

Chronic inflammation has long been thought of as an important risk factor for cancer development. ${ }^{6}$ The present study hypothesized that genetic variations such as SNPs in inflammatory response genes could play a role in influencing the overall inflammatory levels in their carriers, thus conferring them either a risk for, or protection against, prostate cancer. We studied SNPs in the $I L-4, I L-6, I L-8$, and NFKBIA genes and established the relationship between these polymorphisms and prostate cancer risk in the Chinese population.

We showed that the C allele of $I L-4$ rs 2243250 polymorphism could increase the risk of prostate cancer. The $I L-4$ rs2243250 polymorphism is located within a transcription factor-binding site of the gene, which could influence its affinity toward its transcriptional activator, thereby influencing its transcriptional activity. ${ }^{22}$ Several studies have demonstrated that the $\mathrm{C}$ allele of the polymorphism has a lower transcriptional activity compared to the $\mathrm{T}$ allele..$^{23,24}$ Therefore, the $\mathrm{C}$ allele could result in a low-level production of the anti-inflammatory cytokine, which in turn caused the inefficient inflammation inhibitory function, leading to an increased risk of prostate cancer, as in the case of our observation in this study.

In addition, we also demonstrated a reduction in prostate cancer risk by the $\mathrm{C}$ allele of the rs 10499563 polymorphism of $I L-6$. In fact, the role of IL-6 in prostate cancer has been firmly established. It has been shown that IL-6 could promote epithelial-mesenchymal transition and can lead to the malignant transformation of prostate cells. ${ }^{25}$ It has also been observed that high levels of IL-6 and its receptors are produced by cancerous cells of the prostate. ${ }^{26}$ The $\mathrm{C}$ allele of the $I L-6$ rs 10499563 polymorphism has been shown to be associated with a decreased IL-6 concentration, and therefore 
an overall decreased inflammatory level. ${ }^{27}$ This could be the reason why the $\mathrm{C}$ allele was observed to reduce the risk of prostate cancer in our study.

The rs 4073 polymorphism of the $I L-8$ gene has been found to alter the transcriptional activity of the gene. ${ }^{28}$ However, we failed to find any association between the rs4073 polymorphism of $I L-8$ and prostate cancer risk in our study. This finding contradicted with that reported in a recent systematic review and meta-analysis, which showed that the A allele could reduce prostate cancer risk. ${ }^{29}$ The disparity of these findings could be due to the different population investigated in our study and those included in the meta-analysis.

In addition, we found no association of the two NFKBIA polymorphisms, rs2233406 and rs3138053, with prostate cancer risk. It has been shown that the variant alleles of NFKBIA rs2233406 and rs3138053 polymorphisms were associated with a decreased promoter activity of $N F K B I A .{ }^{30}$ As such, the polymorphisms have been reported to be associated with several cancers..$^{21,31,32}$ However, no study has been performed previously to evaluate the association between the two NFKBIA polymorphisms and prostate cancer risk. Our study provides the first evidence of the lack of association between two NFKBIA polymorphisms and prostate cancer risk in the Chinese population.

In conclusion, our study demonstrated that the variant allele of rs2243250 of $I L-4$ and rs 10499563 of $I L-6$ was associated with increased and decreased prostate cancer risk, respectively, in the Chinese population. No association was observed between rs4073 of $I L-8$, as well as rs2233406 and rs3138053 of NFKBIA, and prostate cancer risk in the population. However, studies with a larger sample size are required to confirm our findings.

\section{Acknowledgments}

We thank the nurses in The First Affiliated Hospital of Nanchang University for their help in recruiting the study subjects. We also thank the study subjects for their willingness to participate in this study. No grant was obtained for this study.

\section{Disclosure}

The authors report no conflicts of interest in this work.

\section{References}

1. Ferlay J, Soerjomataram I, Ervik M, et al. GLOBOCAN 2012 v1.0, Cancer Incidence and Mortality Worldwide: IARC Cancer Base No. 11. Lyon, France: International Agency for Research on Cancer; 2013. Available from: http://globocan.iarc.fr. Accessed June 1, 2015.
2. McCracken M, Olsen M, Chen MS Jr, et al. Cancer incidence, mortality, and associated risk factors among Asian Americans of Chinese, Filipino, Vietnamese, Korean, and Japanese ethnicities. CA Cancer J Clin. 2007;57(4):190-205.

3. Cullen J, Elsamanoudi S, Brassell SA, et al. The burden of prostate cancer in Asian nations. J Carcinog. 2012;11:7.

4. Jemal A, Bray F, Center MM, et al. Global cancer statistics. CA Cancer J Clin. 2011;61:69-90.

5. Deutsch E, Maggiorella L, Eschwege P, Bourhis J, Soria JC, Abdulkarim B. Environmental, genetic, and molecular features of prostate cancer. Lancet Oncol. 2004;5(5):303-313.

6. Rakoff-Nahoum S. Why cancer and inflammation? Yale J Biol Med. 2006;79(3-4):123-130.

7. Kirschenbaum A, Klausner AP, Lee R, et al. Expression of cyclooxygenase- 1 and cyclooxygenase- 2 in the human prostate. Urology. 2000;56:671-676.

8. Gupta S, Srivastava M, Ahmad N, et al. Over-expression of cyclooxygenase-2 in human prostate adenocarcinoma. Prostate. 2000; 42:73-78.

9. Mueller E, Smith M, Sarraf P, et al. Effects of ligand activation of peroxisome proliferator-activated receptor gamma in human prostate cancer. Proc Natl Acad Sci U S A. 2000;97:10990-10995.

10. Voronov E, Shouval DS, Krelin Y, et al. IL-1 is required for tumor invasiveness and angiogenesis. Proc Natl Acad Sci U S A. 2003;100: 2645-2650.

11. Lu S, Dong Z. Characterization of TGF-beta-regulated interleukin-8 expression in human prostate cancer cells. Prostate. 2006;66: 996-1004.

12. Kwon EM, Salinas CA, Kolb S, et al. Genetic polymorphisms in inflammation pathway genes and prostate cancer risk. Cancer Epidemiol Biomarkers Prev. 2011;20(5):923-933.

13. Fillon M. Biomarkers and prostate cancer progression. J Natl Cancer Inst. 2011;103(21):1570-1571.

14. Kesarwani P, Ahirwar DK, Mandhani A, Mittal RD. Association between $-174 \mathrm{G} / \mathrm{C}$ promoter polymorphism of the interleukin- 6 gene and progression of prostate cancer in North Indian population. DNA Cell Biol. 2008;27(9):505-510.

15. Volpert OV, Fong T, Koch AE, et al. Inhibition of angiogenesis by interleukin 4. J Exp Med. 1998;188(6):1039-1046.

16. Abdallah A, Sato H, Grutters JC, et al. Inhibitor kappa B-alpha (IkappaB-alpha) promoter polymorphisms in UK and Dutch sarcoidosis. Genes Immun. 2003;4(6):450-454.

17. Huncharek M, Haddock KS, Reid R, Kupelnick B. Smoking as a risk factor for prostate cancer: a meta-analysis of 24 prospective cohort studies. Am J Public Health. 2010;100(4):693-701.

18. Nieters A, Yuan JM, Sun CL, et al. Effect of cytokine genotypes on the hepatitis B virus-hepatocellular carcinoma association. Cancer. 2005;103(4):740-748.

19. Yang L, Sun MJ, Liu JW, Xu Q, Yuan Y. IL-6-6331 (T/C, rs10499563) is associated with decreased risk of gastric cancer in Northern Chinese. Asian Pac J Cancer Prev. 2013;14(12):7467-7472.

20. Vairaktaris E, Yapijakis C, Serefoglou Z, et al. The interleukin-8 $(-251 \mathrm{~A} / \mathrm{T})$ polymorphism is associated with increased risk for oral squamous cell carcinoma. Eur J Surg Oncol. 2007;33(4): 504-507.

21. Tan SC, Suzairi MS, Aizat AA, et al. Gender-specific association of NFKBIA promoter polymorphisms with the risk of sporadic colorectal cancer. Med Oncol. 2013;30(4):693.

22. Tindall EA, Severi G, Hoang HN, et al; Australian Prostate Cancer BioResource. Comprehensive analysis of the cytokine-rich chromosome $5 \mathrm{q} 31.1$ region suggests a role for IL-4 gene variants in prostate cancer risk. Carcinogenesis. 2010;31(10):1748-1754.

23. Tangteerawatana P, Pichyangkul S, Hayano M, et al. Relative levels of IL4 and IFN-gamma in complicated malaria: association with IL4 polymorphism and peripheral parasitemia. Acta Trop. 2007;101(3): 258-265. 
24. Kabesch M, Tzotcheva I, Carr D, et al. A complete screening of the IL4 gene: novel polymorphisms and their association with asthma and IgE in childhood. J Allergy Clin Immunol. 2003;112(5):893-898.

25. Rojas A, Liu G, Coleman I, et al. IL-6 promotes prostate tumorigenesis and progression through autocrine cross-activation of IGF-IR. Oncogene. 2011;30(20):2345-2355.

26. Knüpfer H, Preiss R. sIL-6R: more than an agonist? Immunol Cell Biol. 2008;86(1):87-91.

27. Smith AJ, D'Aiuto F, Palmen J, et al. Association of serum interleukin-6 concentration with a functional IL6 $-6331 \mathrm{~T}>\mathrm{C}$ polymorphism. Clin Chem. 2008;54(5):841-850.

28. Hull J, Thomson A, Kwiatkowski D. Association of respiratory syncytial virus bronchiolitis with the interleukin 8 gene region in UK families. Thorax. 2000;55(12):1023-1027.
29. Wang N, Zhou R, Wang C, et al. -251 T/A polymorphism of the interleukin- 8 gene and cancer risk: a HuGE review and metaanalysis based on 42 case-control studies. Mol Biol Rep. 2012;39(3): 2831-2841.

30. Klement JF, Rice NR, Car BD, et al. IkappaBalpha deficiency results in a sustained NF-kappaB response and severe widespread dermatitis in mice. Mol Cell Biol. 1996;16(5):2341-2349.

31. He Y, Zhang H, Yin J, et al. IkappaBalpha gene promoter polymorphisms are associated with hepatocarcinogenesis in patients infected with hepatitis B virus genotype C. Carcinogenesis. 2009;30(11):1916-1922.

32. Lin CW, Hsieh YS, Hsin CH, et al. Effects of NFKB1 and NFKBIA gene polymorphisms on susceptibility to environmental factors and the clinicopathologic development of oral cancer. PLoS One 2012;7(4):e35078.
OncoTargets and Therapy

\section{Publish your work in this journal}

OncoTargets and Therapy is an international, peer-reviewed, open access journal focusing on the pathological basis of all cancers, potential targets for therapy and treatment protocols employed to improve the management of cancer patients. The journal also focuses on the impact of management programs and new therapeutic agents and protocols on

\section{Dovepress}

patient perspectives such as quality of life, adherence and satisfaction. The manuscript management system is completely online and includes a very quick and fair peer-review system, which is all easy to use. Visit http://www.dovepress.com/testimonials.php to read real quotes from published authors.

Submit your manuscript here: http://www.dovepress.com/oncotargets-and-therapy-journal 